\title{
Decoding the neural representation of fine-grained conceptual categories
}

Marta Ghio ${ }^{1}$, Matilde Maria Serena Vaghi ${ }^{2, *}$, Daniela Perani ${ }^{2,3,4}$, and Marco Tettamanti ${ }^{3,4, \S}$

${ }^{1}$ Institute for Experimental Psychology, Heinrich-Heine-University, Duesseldorf, Germany; ${ }^{2}$ Faculty of Psychology, Vita-Salute San Raffaele University, Milano, Italy; ${ }^{3}$ Nuclear Medicine Unit, San Raffaele Hospital, Milano, Italy; ${ }^{4}$ Division of Neuroscience, San Raffaele Scientific Institute, Milano, Italy.

*M.M.S.V. is now at the Department of Psychology, Behavioural and Clinical Neuroscience Institute, University of Cambridge, UK.

${ }^{\S}$ Corresponding author:

Marco Tettamanti, PhD, San Raffaele Scientific Institute, Via Olgettina 58, I-20132 Milano, Italy.

Email: tettamanti.marco@hsr.it 


\section{Abstract}

Neuroscientific research on conceptual knowledge based on the grounded cognition framework has shed light on the organization of concrete concepts into semantic categories that rely on different types of experiential information. Abstract concepts have traditionally been investigated as an undifferentiated whole, and have only recently been addressed in a grounded cognition perspective. The present fMRI study investigated the involvement of brain systems coding for experiential information in the conceptual processing of fine-grained semantic categories along the abstract-concrete continuum. These categories consisted of Mental state-, Emotion-, Mathematics-, Mouth action-, Hand action-, and Leg action-related meanings. Thirty-five sentences for each category were used as stimuli in a 1-back task performed by 36 healthy participants. A univariate analysis failed to reveal category-specific activations. Multivariate pattern analyses, in turn, revealed that $\mathrm{fMRI}$ data contained sufficient information to disentangle all six fine-grained semantic categories across participants. However, the category-specific activity patterns showed no overlap with the regions coding for experiential information. These findings demonstrate the possibility of detecting specific patterns of neural representation associated with the processing of fine-grained conceptual categories, crucially including abstract ones, though bearing no anatomical correspondence with regions coding for experiential information as predicted by the grounded cognition hypothesis.

Keywords: language; semantic memory; semantic categories; grounded cognition; embodied cognition; experiential knowledge. 


\section{Introduction}

Semantic cognition encompasses the ability to represent and process meanings, and to drive their context-appropriate use in verbal and non-verbal behaviours (Jefferies, 2013). Converging neuropsychological (Lambon Ralph, 2013; Jefferies, 2013) and neuroimaging (Binder et al., 2009) evidence has shown that semantic knowledge draws on heteromodal brain regions, including the anterior temporal lobe and the inferior parietal cortex. The functional role of these regions has been interpreted as either distinct semantic subsystems (Mahon \& Caramazza, 2008) or conceptual hubs supplying modulatory or integration functions (Patterson et al., 2007). In addition, numerous neuropsychological, neuroimaging, and behavioural studies (Binder \& Desai, 2011) have shown that conceptual processing activates distributed cortical regions coding for multimodal (e.g., perceptual, motor, affective) experiential information (henceforth called "experiential brain systems", see Ghio \& Tettamanti, 2015). The interpretation of these experiential brain systems in conceptual processing is controversial, for two main reasons. Firstly, whether experiential brain systems are indeed constitutive to conceptual representations. Secondly, whether this holds true for both concrete and abstract concepts. Concrete concepts (e.g., hammer, cat) are defined as referring to entities that can be experienced through senses or actions. In turn, abstract concepts (e.g., freedom, hope) are defined by exclusion as referring to entities that cannot directly be experienced through senses or actions.

A broad range of theoretical hypotheses relating to perceptual and motor representations have been proposed. At one extreme is the idea that neither concrete nor abstract meanings rely on perceptual and motor representations (Mahon \& Caramazza, 2008). At the other extreme are strong embodiment hypotheses which propose that both abstract and concrete meanings are grounded in sensory and motor systems, which for abstract concepts may occur via a metaphorical extension of concrete concepts (Gallese \& Lakoff, 2005; Gibbs, 2006). Between these proposals are weak embodiment accounts developed within the grounded cognition framework (Barsalou, 2010) which 
suggest that concepts are based, at least in part, on experiential representations reflecting the types of experience engaged during conceptual acquisition (Meteyard et al., 2012; Kiefer \& Pulvermueller, 2012). Importantly, according to the grounded cognition framework, experiential representations do not necessarily refer to sensory and motor information, but may also refer to information about internal states (e.g., interoception, mentalizing, beliefs, affects, self-thoughts, intention recognition) and to information about physical and social contexts (Barsalou, 2010; Borghi \& Binkofski, 2014; Wilson-Mendenhall et al., 2011). According to this perspective, experiential representations, together with the semantic system, play a role in encoding both concrete and abstract concepts, albeit in different proportions. While concrete meanings largely rely on sensory and motor representations, abstract meanings primarily draw on either introspective (Barsalou \& Wiemer-Hastings, 2005), emotional (Kousta et al., 2011; Vigliocco et al. 2014) or social information (Borghi \& Binkofski, 2014). Furthermore, abstract concepts may draw at least in part on linguistic information. Indeed, we mainly learn the meaning of abstract words through language (e.g., by reading a definition in a dictionary or by having the meaning of a word explained verbally; Della Rosa et al., 2010; Granito et al., 2015; Wauters et al., 2003), and we extract several aspects of abstract meanings from verbal associations (e.g., word co-occurrence patterns and syntactic information; Simmons et al., 2008; Vigliocco et al., 2009). Nevertheless, the role of sensory and motor information in abstract meaning representations is not denied, since perceptual experiences and actions may partially constitute the complex situations in which abstract concepts are situated (e.g., the concept of "convince" encompasses listening to and talking to other people; Wilson-Mendenhall et al., 2011).

Until now, evidence about the involvement of the experiential systems in conceptual processing was mainly derived from empirical research on concrete concepts, by adopting a categorical approach. For example, research on object-related knowledge - organized by taxonomic categories (e.g., tools, animals, vegetables) or feature-based categories (e.g., colour, sound, odour, 
functional features, etc.) - lead to the finding of partially segregated, category-specific cerebral networks (for reviews see Martin, 2007; Cappa, 2008). Studies on action semantics (i.e. motorbased knowledge conveyed, for example, by verbs referring to actions performed with mouth, hands, and legs) showed activations in temporal semantic areas, as well as somatotopically organized activations in fronto-parietal motor circuits (Grezes \& Decety, 2001; Tettamanti et al., 2005; Saccuman et al., 2006; Pulvermueller, 2013). Even if such findings appear to argue against a barely epiphenomenal role of sensory-motor representations, evidence about their necessary role in conceptual processing is controversial. For example, neuropsychological evidence suggests that impairments of the sensory-motor system result in impoverished rather than fully compromised concept processing (Kemmerer, 2015), or even that these impairments can leave the processing of action verbs unaffected (Papeo \& Hochmann, 2012).

Abstract knowledge, in turn, has been largely overlooked. Evidence for a greater involvement of left-hemispheric perisylvian brain regions in abstract concept processing comes mainly from studies contrasting abstract concepts - considered as an undifferentiated whole - with concrete concepts (for a review, see Wang et al., 2010). These results have been explained in light of traditional accounts by which abstract meanings rely only on verbal representations, and lack any direct access to sensory-perceptual representations (Dual Coding theory: Paivio, 1971; 2013; Context Availability theory: Schwanenflugel et al., 1988). However, closer examination of the multiple nature of abstract knowledge showed that abstract meanings, when compared to concrete meanings, are more related to affective, emotional, and social features (Kousta et al., 2011; Troche et al., 2014), and are associated with a broader variability of contexts and situations (Hoffman et al., 2013). Some functional magnetic resonance imaging (fMRI) studies revealed that the processing of abstract concepts elicited greater activity in the retrosplenial cingulate cortex, as a semantic region relevant for contextual information coding and introspective state monitoring (Tettamanti et al., 2008; Ghio \& Tettamanti, 2010), as well as the anterior cingulate cortex, a brain region plausibly 
involved in affective information processing (Vigliocco et al., 2014).

Crucially, a paradigm shift in the experimental investigation of abstract knowledge is now emerging within the grounded cognition framework. This suggests taking into account fine-grained categories with an abstract content, by analogy to what has been done for categories with concrete content. Although the classification of abstract meanings into fine-grained categories is less straightforward than that relating to concrete meanings (Crutch \& Warrington, 2005), it appears to be both theoretically and psychologically plausible (Hampton, 1981; Wiemer-Hastings \& Xu, 2005). Just as with specific concrete categories, a particular pattern of experiential representations might be more or less relevant for each abstract category, depending on the type of characterizing experience (Wilson-Mendenhall et al., 2013; Ghio \& Tettamanti, 2015). For example, the processing of social concepts (e.g., honour, to convince) might encompass brain networks involved in social cognition, whereas mental state concepts (e.g., inference, to think) might rely on representations of introspective states (such as thoughts, beliefs, self-reflection). Similarly, the processing of emotion concepts (e.g., sadness, to enjoy) and mathematics concepts (e.g., arithmetic, to sum) might draw on representations of affective states and numbers/quantities, respectively. Sensory and motor information might also differently contribute to the representation of each abstract meaning.

Psycholinguistic research (Altarriba, et al., 1999; Ghio et al., 2013) revealed that abstract categories related to mental states, emotions, and mathematics are associated with characteristic patterns of psycholinguistic variables. For example, compared with other abstract meanings, emotion words were judged on seven-point Likert scales as more imageable but less concrete and lower in context availability (Altarriba et al., 1999). By using seven-point Likert scales testing the involvement of actions performed with different body-parts in meaning representation, Ghio et al. (2013) showed that emotion-related sentences were relatively more associated with mouth movements than mental state- or mathematics-related sentences (but less than mouth-related 
sentences). A recent neuropsychological study showed a category-specific effect, with emotion meanings preserved in patients with Alzheimer's disease, and social meanings selectively impaired in patients with the semantic variant of primary progressive aphasia (Catricalà et al., 2014).

Consistent with the category-specific grounded cognition hypothesis, fMRI studies showed that abstract social concepts elicited activations in brain regions typically implicated in social cognition, including the temporal pole, the medial prefrontal cortex, the posterior superior temporal sulcus, the precuneus/posterior cingulate, and the fusiform gyrus (Simmons et al., 2009; Zahn et al., 2007). An fMRI study by Moseley et al. (2012) focused on emotion concepts, distinguishing between concrete, action-related (e.g., frown) and abstract, action-unrelated (e.g., ail) emotion words. The results revealed that silent-reading of both types of emotion words activated not only emotion brain regions, but also sensorimotor areas. Limited neuroimaging research, however, has so far addressed the grounded cognition hypothesis with respect to other abstract categories, comparing them in a systematic way. To our knowledge, only one fMRI study until present has directly contrasted social and mathematics-related abstract concepts, though limited to one single experimental word for each category (Wilson-Mendenhall et al., 2013). The results showed that brain regions underlying numerical cognition (e.g., bilateral intraparietal sulcus) were active during the repeated presentation of the word arithmetic, whereas brain regions underlying social cognition (e.g., medial prefrontal cortex) were active during the repeated presentation of the word convince.

The present fMRI study systematically investigated the involvement of brain systems coding for experiential information in the conceptual processing of six fine-grained semantic categories characterized at the psycholinguistic level in our previous rating study (Ghio et al., 2013). Among the meanings traditionally considered as abstract (Paivio, 1971), we distinguished between three categories: Mental state-related (e.g., Lei ricorda il passato (She remembers the past)), Emotionrelated (e.g., Lei prova il disgusto (She feels the disgust)), and Mathematics-related (e.g., Lei calcola la somma (She calculates the sum)). Three concrete categories were also investigated: 
Mouth action-related (e.g., Lei morde il frutto (She bites the fruit)), Hand action-related (e.g., Lei taglia la carota (She cuts the carrot)), and Leg action-related (e.g., Lei calcia la palla (She kicks the ball)).

First, we applied a univariate analysis in order to test the grounded cognition hypothesis with respect to each semantic category, namely: (i) Mental state-related meanings activate the default mode network associated with self-reflection, mind wandering, and introspective states (Spreng et al., 2009); (ii) Emotion-related meanings activate the neural network involved in processing emotions, and possibly also motor regions (Moseley et al., 2012; Tettamanti et al., 2012); (iii) Mathematics-related meanings activate a network of parietal and frontal cortical areas involved in calculation and in the representation of numbers and quantities (Nieder \& Dehaene, 2009); (iv) concrete, Mouth action-, Hand action-, and Leg action-related meanings activate the somatotopically organized action-specific fronto-parietal network (Tettamanti et al., 2005).

Second, we applied multivariate pattern analysis (MVPA) (Haynes \& Rees, 2006; Kriegeskorte, 2011; O'Toole et al., 2007; Pereira et al., 2009) to test whether the fMRI data contained enough information to distinguish between the six specific categories, in so far as they might be subserved by (at least partially) segregated representations. Multivariate analysis has been successfully used for investigating semantic processes. Classification studies on object categories showed that, irrespective of the modality of presentation (words, pictures, sounds), different object categories can be distinguished, such as, faces, houses, cats, bottles, scissors, shoes, chairs (Haxby et al., 2001), animals, and tools (Simanova et al., 2014; for reviews, see Vindiola \& Wolmetz, 2011; Kriegeskorte, 2011). Consistent with a grounded cognition perspective, Buchweitz et al. (2012) demonstrated that essential voxels for classifying tools and dwellings were located, respectively, in brain regions associated with encoding motor/manipulation semantic properties (postcentral gyri and inferior parietal lobule) and shelter properties (parahippocampal gyrus). Up to now, little evidence has been provided about abstract categories. A recent study applied multivariate analysis 
to discriminate between abstract properties of mentally simulated situations (e.g., Attributes, Communications, Events, Social Roles). The results revealed that the investigated abstract properties could be reliably discriminated, though only within participants (Anderson et al., 2014). Using multivariate analyses, the present study aimed at extending previous research by considering fine-grained categories not investigated so far.

In order to provide independent anatomo-functional specifications for the grounded cognition hypothesis, we carried out a functional MRI localizer study in a separate group of participants, including six independent functional localizer tasks targeting the extensive functional brain systems activated by direct experience with the conceptual referents of each semantic category (Mental state localizer; Emotion localizer; Mathematics localizer; Mouth, Hand and Leg movement localizers). This functional localization approach was aimed at testing, from a grounded cognition perspective, the involvement of brain systems participating to different aspects of conceptual experience in linguistic semantic processing. In the univariate analysis, localizer data were used in order to restrict hypothesis testing to specific regions of interest (ROI). In multivariate analyses, a machine learning classifier was trained to distinguish between the activation patterns of the six different localizer tasks. The trained classifier was then tested on its ability to discriminate patterns of neural activity elicited by sentence processing of the six different semantic categories. 


\section{Materials and methods}

\subsection{Participants}

Thirty-six participants $(18 \mathrm{M} / 18 \mathrm{~F}$; mean age 21.16 years, $S D=3.87)$ of comparable education level volunteered in the experiment. All participants were right-handed (mean score $0.96, S D=0.04$ ) according to the Edinburgh Inventory (Oldfield, 1971). All reported no history of neurological, psychiatric, developmental diagnoses, head or brain injury or trauma, and no sensory or perceptual difficulties other than corrected vision. Participants reported that they did not take medications affecting the nervous system. They gave written consent to participate in the study after receiving a careful explanation of the procedures. The study was approved by the Ethics Committee of the San Raffaele Scientific Institute, Milano, Italy.

\subsection{Experimental design and stimuli}

The experimental design included one factor, i.e. Semantic category, with six experimental conditions: Mental state- (Ms), Emotion- (Em), Mathematics- (Ma), Mouth action- (Mo), Hand action- (Ha), and Leg action- (Le) related semantic categories.

Each of the six semantic categories included 35 Italian sentences. A comprehensive characterization of sentence stimuli is provided in Ghio et al. (2013). Here, we only report the details relevant for the present study. All the 210 sentences consisted of four words and had the same syntactic structure: the third person feminine pronoun 'Lei' (Engl. She), and a verb in thirdperson singular, simple present tense, matched to a syntactically and semantically congruent object complement (see examples in the Introduction). All the six categories were matched with respect to (i) linguistic parameters: number of words (all 4-word sentences); number of syllables (Chi-square $(25)=36.371, \mathrm{P}=0.066)$; number of letters $(\mathrm{F}(5,204)=1.250, \mathrm{P}=0.287)$; lexical frequency for, respectively, nouns $(\mathrm{F}(5,204)=1.861, \mathrm{P}=0.103)$, verbs $(\mathrm{F}(5,204)=1.723, \mathrm{P}=0.131)$, and nounverb combinations $(\mathrm{F}(5,204)=1.824, \mathrm{P}=0.110)$; (ii) auditory parameters: intensity $(\mathrm{F}(5,204)=$ 
$1.465, \mathrm{P}=0.203)$, pitch $(\mathrm{F}(5,204)=1.433, \mathrm{P}=0.214)$, duration $(\mathrm{F}(5,204)=2.259, \mathrm{P}=0.050)$. In addition, sentences were rated along the following dimensions: category-specific association (only Ms, Em, Ma categories); body-part association; concreteness, context availability and familiarity (Ghio et al., 2013, Tables 2, 3, 4). The complete set of written and auditory Italian sentences, along with the rating data, can be obtained by sending requests to M.T. (tettamanti.marco@hsr.it).

\subsection{Experimental procedure}

The participants were instructed to carefully listen to all sentences administered via high-quality MRI-compatible headphones, controlled by an optical audio-control unit (MR Confon GmbH, Magdeburg, Germany). The software package Presentation 14.9 (Neurobehavioral Systems, Albany, CA, USA) was used for stimuli presentation. Two seconds after the end of each auditory sentence, either a fixation cross or a question mark was visually presented for 500 ms. If a fixation cross appeared on the screen, the participants were not asked to perform any tasks (experimental trials). If a question mark appeared on the screen, a written sentence was presented for $1000 \mathrm{~ms}$, and the participants were asked to perform a cross-modal 1-back task, i.e. to judge whether the written sentence matched the auditory sentence presented immediately before (catch trials). An overt response was provided by the participants, by slowly blinking their eyelids: either once, if written and auditory sentences were identical (match catch trials), or twice, if written and auditory sentences did not match (mismatch catch trials). Eyelid blinking was chosen in order to avoid confounds with the hypothesized somatotopic motor system involvement induced by the processing of mouth-, hand-, and leg-related meanings. The eyelid blinking responses were video recorded and subsequently scored by a judge (M.G.) and confirmed by an external independent judge (Cohen's kappa $=.979, \mathrm{P}<.001)$.

The stimuli used in the catch trials were 4-word sentences of the same form as the experimental stimuli. In order to avoid shallow sentence processing due to highly predictable mismatches between auditory and written sentences, we introduced variations along the following dimensions: 
(i) verb mismatches (e.g., auditory sentence: She learns the doctrine; written sentence: She explains the doctrine); (ii) object complement mismatches (e.g., auditory sentence: She instils the joy; written sentence: She instils the warmth); (iii) both verb and object complement mismatches (e.g., auditory sentence: She resets the calculator; written sentence: She reads the book).

Before the experimental sessions, the participants completed a brief training to familiarize with the task. Training sentences (11 in total, including 1 match and 1 mismatch catch trial) were related to social, medical, and law semantic categories, in order to avoid contamination effects with the actual experimental stimuli. The training was conducted in the MR scanner with ongoing fMRI data acquisition, in order for the participants to get accustomed to auditory sentence processing with the background acquisition noise.

All participants were presented with all 210 sentences. The stimuli were presented in a jittered event-related mode, divided in three separate fMRI runs, each including a minimum of 11 and a maximum of 12 sentences per experimental condition. Each run was constituted by 89 randomized trials: 68 experimental trials, 12 catch trials, and 9 null trials. The order of the inter-trial intervals was determined by OPTseq2 (surfer.nmr.mgh.harvard.edu/optseq/) so as to maximize the hemodynamic signal sensitivity of the event-related design. Three inter-stimulus interval durations were used, corresponding to $3000 \mathrm{~ms}, 5000 \mathrm{~ms}$, and $7000 \mathrm{~ms}$ (in proportion 4:2:1). The order (across trials) of the within-trial intervals (i.e., both the interval between the auditory sentence and the fixation cross/question mark, and the interval between the question mark and the written sentence) were determined by OPTseq 2 as well. In both cases, three interval durations were used, corresponding to $400 \mathrm{~ms}, 500 \mathrm{~ms}$, and $600 \mathrm{~ms}$ (in proportion 1:1:1).

\subsection{Data acquisition}

MRI scans were acquired with a 3 Tesla Philips Achieva whole body MR scanner (Philips Medical Systems, Best, NL) using an eight-channel Sense head coil (Sense reduction factor $=2$ ). Wholebrain functional images were obtained with a $\mathrm{T} 2 *$-weighted gradient-echo, EPI pulse sequence, 
using BOLD contrast (Repetition Time $(\mathrm{TR})=2000 \mathrm{~ms}$, Echo Time $(\mathrm{TE})=30 \mathrm{~ms})$. Each functional image comprised 31 contiguous axial slices ( $3.4 \mathrm{~mm}$ thick, $0.6 \mathrm{~mm}$ gap), acquired sequentially from bottom to top (field of view $=240 \times 240 \mathrm{~mm}$, matrix size $=96 \times 96$ ). Each participant underwent three consecutive functional scanning sessions on the same day. Each scanning session comprised 340 scans, preceded by 5 dummy scans that were discarded prior to data analysis, and lasted 11 minutes and 30 seconds.

A high-resolution T1-weighted anatomical scan (three-dimensional spoiled-gradient-recalled sequence, 200 slices, $\mathrm{TR}=7.2 \mathrm{~ms}, \mathrm{TE}=3.5 \mathrm{~ms}$, slice thickness $=1 \mathrm{~mm}$, in-plane resolution $1 \mathrm{x} 1$ $\mathrm{mm}$ ) was acquired for each participant.

\subsection{Univariate General Linear Model analysis}

Imaging data were processed using SPM8 (www.fil.ion.ucl.ac.uk/spm). The New Segment procedure was used to segment the structural MRI images of each participant, using a custom template based on a sample of 317 images of healthy adult subjects acquired with the same MR scanner used for the present experiment. The custom template was previously normalized to approximate the Montreal Neurological Institute (MNI) standard space. The images underwent a very light bias regularization and were spatially normalized using an affine spatial normalization. A first New Segment iteration yielded a bias corrected version of each structural image, which was then input in a second New Segment iteration in order to improve the accuracy of the final normalization.

For the purpose of using the resulting segmented structural images of each participant as customized segmentation priors in the New Segment procedure applied to the functional images, the segmented structural images were re-sampled with a spatial resolution of $2 \times 2 \times 4 \mathrm{~mm}$ and smoothed with a 2-mm FWHM Gaussian kernel. Functional images were corrected for slice timing, and realigned to the first scan of the first session acquired for each participant. The images were normalized into the approximate MNI space using the New Segment procedure with subject- 
specific customized structural segmentation priors, and subsequently spatially smoothed with a 6mm FWHM Gaussian kernel. General Linear Model statistical analysis was used. We adopted a two-stage random-effects approach to ensure generalizability of the results at the population level (Penny \& Holmes, 2003). The statistical analysis was restricted to an explicit mask including only the voxels with grey matter tissue probability $>0.1$, based on the re-sampled and smoothed, segmented structural images of each participant.

First-level General Linear Models. The time series of each participant were high-pass filtered at $128 \mathrm{~s}$ and pre-whitened by means of an autoregressive model AR(1). No global normalisation was performed. Hemodynamic evoked responses for all experimental conditions were modelled as canonical hemodynamic response functions. For each participant, we modelled three separate sessions, each with Ms, Em, Ma, Mo, Ha, and Le regressors of evoked responses, aligned to the onset of each auditory sentence. Separate regressors modelled catch trials, aligned to the onset of the written sentence, task instructions, and movement parameters. For the purpose of the secondlevel analysis we defined a set of $t$-contrasts with a weight of +1 for a particular regressor of interest and a weight of zero for all the other regressors, thus resulting in one contrast per experimental condition (respectively, [Ms], [Em], [Ma], [Mo], [Ha], [Le]) for each participant.

Second-level General Linear Model. Using the first-level contrasts, we specified a flexible factorial design. The model included a subject factor (36 levels = 36 participants; independence and equal variance assumed between-subjects) and the Semantic category factor (6 levels = Ms, Em, Ma, Mo, Ha, Le; dependence and equal variance assumed between levels).

We investigated the condition-specific effects for each semantic category by assessing the conjunction null hypothesis (Nichols et al., 2005) for the five pairwise comparisons between that category and each of the other five categories (e.g., for Ms, we calculated the conjunction null of: [Ms - Em], [Ms - Ma], [Ms - Mo], [Ms - Ha], [Ms - Le]). The significance threshold was declared at cluster-level $\mathrm{P}<0.05$, using a Family Wise Error (FWE) type correction for multiple comparisons 
at the whole brain level, based on a pre-specified peak-level uncorrected $\mathrm{P}<0.001$. In order to test the activation of specific brain regions not surviving a whole brain correction, but which were predicted on the basis of the fMRI localizer tasks, we applied a Small Volume Correction (SVC) (P $<0.05$, FWE corrected) (Poldrack et al., 2011). This procedure reduces the stringency of the correction for multiple comparisons by limiting the search for activations to specific brain regions (Poldrack et al., 2011). The small search volumes consisted of ROI defined on the basis of the localizer tasks (see section 2.7).

\subsection{Multivariate pattern analyses}

In order to cope with the problems posed by jittered event-related fMRI designs in MVPA, we performed the analysis on summary GLM beta images, instead of fMRI time series images (Schrouff et al., 2013). For this purpose, and in order to take advantage of voxel-level spatially resolved sensitivity of MVPA, we repeated the SPM8 univariate first-level GLM analysis on unsmoothed fMRI data. The resulting beta images of all participants were merged into a single 4D image file ( 1 beta image per each of the 6 experimental conditions, per each of the 3 fMRI runs, i.e. 18 beta images x 36 participants). The 4D beta image file was loaded into PyMVPA 2.2 software (www.pymvpa.org; Hanke et al., 2009) running under Python 2.7.5 (www.python.org). The 4D image was masked by the SPM8 implicit mask image of the 2nd-level group random-effects flexible factorial analysis (section 2.5), to ensure that the voxels included in the MVPA contained sufficient BOLD signal change across all participants. This implicit mask is essentially a structural mask, reflecting the inclusion of voxels with grey matter tissue probability $>0.1$ in first-level GLM analyses (section 2.5). Z-score normalization of each voxel for each run was performed in order to control for global variations of the hemodynamic response across runs and subjects. No linear detrending was applied, given that the beta images were already corrected for temporal autocorrelations. We used the LinearCSVMC (C-SVM classifier using linear kernel) as implemented in PyMVPA as a Support Vector Machine classification algorithm. 


\subsubsection{Multivariate classification of fine-grained categories}

We investigated whether the fMRI data contained sufficient information to predict the processing of each and every semantic category (Ms, Em, Ma, Mo, Ha, Le). For the purpose of this whole brain joint metalinear SVM classification, the beta images of each participant were averaged across the three runs according to the experimental conditions. This procedure was adopted in order to reduce the intra-subject variability and improve the signal to noise ratio of the data (Quadflieg et al., 2011; Pereira et al., 2009). The multi-way classification was performed in PyMVPA, which provides a framework to create meta-classifiers, among others for the LinearCSVMC classifier used here (Hanke et al., 2009).

Between-subjects analysis was performed using a leave-one-subject-out cross validation procedure in which the LinearCSVMC was trained on data from 35 subjects and then tested on data from the $36^{\text {th }}$ subject. The procedure was repeated 36 times, leaving each subject out once. We report the classification accuracy as the simple mean of the 36 classification results ( 1 for correct; 0 for incorrect) (Akama et al., 2012; Kaplan \& Meyer, 2012), together with the confusion matrix of predicted against actual classes. This cross-individual analysis was performed in order to verify whether information is represented in the same way across individuals. This procedure is analogous to standard analyses that treat subjects as random factor in order to generalize the results at the population level.

Two leave-one-subject-out classification analyses were performed by using, respectively: (i) all brain mask voxels; (ii) a subset of voxels selected through a recursive feature (i.e. voxel) elimination algorithm, yielding a sensitivity analysis (Hanson \& Halchenko, 2008). Recursive feature elimination was performed strictly on the leave-one-subject-out training data, by iteratively eliminating the less sensitive $50 \%$ of voxels, and then selecting the reduced brain voxel partition having the greatest sensitivity. This reduced voxel partition was then used for calculating the classification accuracy on the leave-one-subject-out test data. We report the mean cross-individual 
classification accuracy.

We verified whether the classification accuracy was significantly above the chance-level of $16.6 \%$ (i.e. $100 \%$ / 6 classes). To estimate how likely was the classifier capable of discriminating all or a subset of stimulus categories from each other, we adopted the approach proposed by Olivetti et al. (2012) and implemented in PyMVPA, consisting of a Bayesian hypothesis testing in order to evaluate the posterior probability of each possible partitioning of distinguishable subsets of test classes. For example, in the case of just three classes, the possible partitions of test classes are $[1][2][3] ;[1,2][3] ;[1,3][2] ;[1][2,3] ;[1,2,3]$. Each of these partitions is assigned a posterior probability, where a probability in excess of $1 / \mathrm{K}$ (where $\mathrm{K}$ is the number of hypotheses) would be seen as informative evidence. The output of this analysis is the most likely hypothesis (i.e. partition) to explain the classification confusion matrix.

To further investigate the specificity of each semantic category, we ran separate SVM classifications on each and every pair of categories (i.e. Ms-Em, Ms-Ma, Ms-Mo, Ms-Ha, Ms-Le, Em-Ma, Em-Mo, Em-Ha, Em-Le, Ma-Mo, Ma-Ha, Ma-Le, Mo-Ha, Mo-Le, Ha-Le). Overall maximum cross-individual sensitivity weights - representing the extent to which each voxel contributes to the separation of one category from the other in the test data - were calculated, yielding a sensitivity map for each between category pair classification. In order to obtain spatially localized sensitivity information for each category, we calculated the intersection of the five sensitivity maps representing a specific category compared with each of the other five categories (e.g., for Ms, we calculated the intersection of the following sensitivity maps: Ms-Em, Ms-Ma, MsMo, Ms-Ha, Ms-Le). Light smoothing of the sensitivity maps with a 3-mm FWHM Gaussian kernel was applied before the calculation of the intersection.

\subsubsection{Multivariate classification across studies}

We also performed a set of between-subjects MVPA across studies, consisting of SVM classification of the functional data across the fMRI localizer study (section 2.7) and the sentence 
processing study. Such analyses were carried out in order to examine whether localizer activation patterns for the actual experience of mental states, the actual processing of emotion pictures and of mathematical quantities, and the actual movements of mouth, hands, or legs could specifically predict the activation patterns induced by the processing of the six respective sentence categories. The functional localizer beta images were considered as training data, whereas the linguistic beta images were considered as testing data. The multi-way classification was performed by applying the meta LinearCSVMC classifier in PyMVPA. A between-subjects, leave-one-subject-out cross validation procedure was applied (training: data of 48 subjects in the localizer study; testing: repeated 36 times, each time taking the data of one of the 36 subjects in the linguistic study). The classification analysis was performed by using all brain mask voxels. We report the results of Bayesian hypothesis testing, with which we evaluated the posterior probability of each possible partitioning of distinguishable subsets of test classes.

\subsection{Functional MRI localizer study}

Fifty participants $(25 \mathrm{M} / 25 \mathrm{~F}$; mean age 23.02 years, $\mathrm{SD}=4.88)$ of comparable education level volunteered for the experiment. All participants were right-handed (mean score 0.94, SD $=0.05$ ) according to the Edinburgh Inventory. The same inclusion/exclusion criteria and ethics procedure were applied as for the main study. Two subjects $(1 \mathrm{M} / 1 \mathrm{~F})$ were excluded due to an incidental finding of structural brain anomalies.

MRI data were acquired on the same scanner and with the same parameters as for the main study, except that whole-brain functional images were acquired with a TR $=3000 \mathrm{~ms}$, and comprised 35 contiguous axial slices (3.2 mm thick, $0.8 \mathrm{~mm}$ gap), acquired sequentially from bottom to top (field of view $=240 \mathrm{~mm} \times 240 \mathrm{~mm}$, matrix size $=128 \times 128)$. Each participant underwent six consecutive localizer scanning sessions on the same day. Each localizer session comprised 56 scans, preceded by 5 dummy scans that were discarded prior to data analysis.

We followed the same image processing pipeline using SPM8 as for the sentence processing study. 
The Mental state localizer was analysed using Independent Components Analysis, whereas the remaining localizer tasks were analysed using a univariate General Linear Model (Supplementary information SI.1). The activation networks identified by means of the localizer tasks were used for the specification of ROI for the SVC analysis of the six fine-grained semantic categories (Supplementary information SI.2).

Mental state localizer. A resting state localizer was performed. Subjects laid still in the scanner with their eyes closed for three minutes. The Mental state localizer activated a bilateral network of brain regions including, among other regions, areas of the Default Mode Network (Figure S1A, Table S1A), consistently with the resting state literature (for a review, see Fox \& Raichle 2007).

Emotion localizer task. A rest block during which the word "Rest" appeared on the screen (duration $=24000 \mathrm{~ms}$ ) was alternated three times with a task-related block during which participants were visually presented with pictures of human faces with joyful, angry, and neutral expressions in semirandomized order. Stimuli were selected from the NimStim Emotional Face Database (Tottenham et al., 2009). Each block (24000 ms) included 8 pictures, each lasting $3000 \mathrm{~ms}$. Participants were required to carefully observe each picture without performing any additional tasks. The Emotion localizer task activated a brain circuit including, among other regions, the fusiform gyrus, the amygdala, the hippocampus, the superior medial frontal gyrus, bilaterally (Figure S1B, Table S1B), in agreement with previous evidence on emotional facial expression processing (for a review, see Fusar-Poli et al., 2009).

Mathematics localizer task. A rest block during which the word "Rest" appeared on the screen (24000 ms) was alternated three times with a task-related block during which participants covertly performed mathematical calculations on visually presented numbers and operators. Each block started with the word "Count!" (2500 ms) followed by a series of calculations, such as for example “47” (2500 ms), “+ 13” (5500 ms), “x 2” (5500 ms). Finally, an equal mark and a question mark “= ?" appeared on the screen $(5500 \mathrm{~ms})$. Participants were required to carry out each step in the 
intermediate mental calculations, until achieving the final result. The Mathematics localizer task elicited activations in, among other regions, bilateral frontal and parietal cortices. In particular, we found activations in brain regions associated to the processing of numerical quantities and calculations (for a review, see Pinel et al., 2007), such as the middle frontal gyrus and the intraparietal sulcus (Figure S1C, Table S1C).

Body-part movement localizer tasks. To localize motor cortex activation corresponding to mouth, hand, and foot movements, participants were asked to perform three different motor tasks. In each localizer task, a rest block during which the word "Rest" appeared on the screen (24000 ms) was alternated three times with a movement block $(24000 \mathrm{~ms})$ during which the sentence "Move your tongue / right hand / right foot" remained visible on the screen, and participants had to execute the corresponding movement at a constant pace. Tongue movements consisted of continuous tongue rotation. Hand movements consisted of opening and closing the right hand fingers, once every 1-2 seconds. Leg movements consisted of alternated ankle dorsiflexion and plantar flexion of the right foot, once every 1-2 seconds. The Body-part movement localizers activated extended brain networks including the motor and premotor cortices (Figure S1D, Table S1D-F), in agreement with previous findings for mouth, hand, and leg movements (Tomasino et al., 2010; Carota et al., 2012; Moseley et al., 2012). 


\section{Results}

\subsection{Behavioural results}

The videos with the participants' eyelid blinking responses in the fMRI linguistic study showed that, qualitatively, all participants maintained an adequate alert state throughout the cross-modal 1back task. Their overall mean accuracy was $96.88 \%$ (range 50\%-100\%, one outlier subject, see Supplementary information SI.3). The mean accuracy did not differ across runs $(\mathrm{F}(2,104)=0.355, \mathrm{P}$ $=.702 ;$ run $1: M=97.92 \%, S D=8.54 \% ;$ run $2: M=96.19 \%, S D=9.34 \%$, values from one subject are missing due to technical errors; run $3: M=96.53 \%, S D=9.63 \%$ ).

\subsection{Univariate General Linear Model analysis}

In the univariate General Linear Model analysis, we found no significant activations for any of the six semantic categories. No specific activations were found even in a priori specified brain regions based on the functional localizer tasks.

Since these negative results could be due to inefficiency of the fMRI event-related design, we performed two control analyses to ascertain whether there was sufficient trial- and stimulus-related signal detection in our data.

\subsubsection{Control analysis of activation induced by all experimental trials}

We explored the pattern of activation for the experimental trials (i.e. trials in which subjects linguistically processed sentences belonging to the six semantic categories) compared with catch trials (i.e. trials in which subjects performed the cross-modal 1-back task) (Supplementary information SI.4). We found bilateral clusters of activation in the superior temporal gyrus, Heschl's gyrus, temporal pole, precentral gyrus, postcentral gyrus, paracentral lobule, and middle/posterior cingulate gyrus (Table S2). Additional activation foci were located in the right inferior frontal gyrus (pars triangularis) and in the left middle temporal gyrus.

\subsubsection{Control analysis for the abstract-concrete distinction}


We collapsed the six categories as either abstract (Ms, Em, Ma) or concrete (Mo, Ha, Le) for comparison with well-established activation patterns associated to the abstract-concrete distinction (Supplementary information SI.5). The Abstract > Concrete contrast revealed that abstract sentences elicited greater activations in the left temporal pole, and the left inferior parietal lobule, as well as bilateral activations in the superior and middle temporal gyri (Figure S2A, Table S3A). In addition, by applying SVC, we found a significant cluster of activation in the inferior frontal gyrus (pars triangularis). The Concrete $>$ Abstract contrast yielded activation increases in a left fronto-parietal network, including the inferior frontal gyrus (pars orbitalis and pars triangularis), and the inferior parietal lobule (Figure S2B, Table S3B; all the significant p-values were obtained through SVC).

\subsection{Multivariate classification of fine-grained categories}

The six-way discrimination over all brain voxels yielded a mean classification accuracy of $37.0 \%$ across subjects, as compared to a chance level of $16.6 \%$. Recursive feature elimination yielded an increase of the mean classification accuracy (41.7\%). Examination of the confusion matrix (Figure 1A) revealed that the six categories were successfully classified $($ Chi-square $=$ $\left.168.00, \mathrm{P}=2.7 * 10^{-19}\right)$. Bayesian analysis of the confusion matrix revealed that the most likely partition was $[\mathrm{Ms}],[\mathrm{Em}],[\mathrm{Ma}],[\mathrm{Mo}],[\mathrm{Ha}],[\mathrm{Le}]$ (Bayes Factor $>1$ with respect to all other possible partitions). These results suggest that our fMRI data set contains enough information to fully discriminate between the six semantic categories, although with weak evidence with respect to the $2^{\text {nd }}$, the $3^{\text {rd }}$, and the $4^{\text {th }}$ ranked partitions (Table 1 ).

The MVPA classification applied on each and every pair of categories revealed that for each pair, the mean classification accuracy was above the chance threshold, with the exception of the Ha-Le pair which attained a close to significance classification accuracy $(\mathrm{P}=0.065)$ (Table 2). Crucially, for each pair of categories, including Ha-Le, the Bayesian analysis of the confusion matrix revealed that the two categories could be separated (Table 2). For each category, we 
calculated the intersection of the five sensitivity maps between that particular category and each of the other five categories. The sensitivity regions specific to each of the six categories were sparsely distributed over the sensory and heteromodal cortices and over the subcortical nuclei of both hemispheres (Figure 1B, Table S4). Notably, there were a few anatomical regions that presented sub-parcels specific to each of the six categories, such that, as a whole, they contributed to distinguish all the categories from each other. These were the left inferior frontal gyrus (pars triangularis and pars orbitalis) and, bilaterally, the insula lobe.

\subsection{Multivariate analysis across studies}

We performed a six-way discrimination of functional data across the localizer task study and the sentence processing study. The Bayesian analysis of the resulting confusion matrix revealed that the most likely partition was ([Ms, Em, Ma, Ha, Le], [Mo]). This result suggested that the data of the localizer tasks did not contain matching information capable of discriminating between the six semantic categories. 


\section{Decoding fine-grained conceptual categories}

\section{Discussion}

The present work tested the grounded cognition hypothesis that brain regions coding for experiential information support the semantic processing of fine-grained conceptual categories along the abstract-concrete continuum, by applying either univariate or multivariate analyses. The univariate standard analysis failed to reveal specific brain activations for the fine-grained semantic categories. Crucially, however, by applying MVPA we demonstrated that the neural activity underlying sentence processing contained sufficient information to successfully discriminate between all the six semantic categories, and that the fine-grained category-specific activity patterns were consistent across participants. This was demonstrated most clearly by the SVM classification applied on each and every pair of fine-grained categories: Bayesian testing provided evidence that the two categories in each pair could be well separated (Table 2). Finally, the MVPA classification of functional data across the localizer task study and the sentence processing study showed no overlap between category-specific activity patterns and the regions coding for experiential information, contrary to the grounded cognition hypothesis.

The results of the multivariate analysis extend previous evidence showing the possibility of discriminating between different types of object categories (for reviews, see Vindiola \& Wolmetz, 2011; Kriegeskorte, 2011; Kaplan \& Meyer, 2012), by investigating abstract-related meanings not considered so far in a specific and systematic way. Only recently, scholars within the grounded cognition framework have recognized the importance of extending the research to the domain of abstract meanings and thus little evidence is available. Anderson et al. (2014) reported results indicating that WordNet taxonomic categories for abstract properties of mentally simulated situations could be distinguished, although only within subjects. Compared with Anderson et al. (2014), the results of the present study indicate that it is possible to identify across subjects the neural representations associated with different semantic categories along the abstract-concrete continuum. 
An important point of discussion regards the different outcomes of the univariate and multivariate analyses. Indeed, we found evidence that, contrary to univariate analysis, multivariate analysis techniques can reveal the existence of categorically organized information in the patterns of fMRImeasured brain activation along fine-grained semantic categories. This outcome discrepancy may reflect the different scopes of the two analysis approaches. Multivariate analysis is sensitive to the distributed coding of information, whereas univariate analysis is more sensitive to global activations (Haynes \& Rees, 2006; Kriegeskorte, 2011; O’Toole et al., 2007; Pereira et al., 2009). As such, a null result in the univariate analysis cannot rule out the possibility that specific distributed activation patterns subserving the semantic processing of these categories can be detected using the multivariate analysis. Compared to univariate analysis, MVPA can perform signal detection in finegrained patterns across multiple voxels, even when the average activations within these voxels are not significantly different (Norman et al., 2006; Mur et al., 2009). Our results of the multivariate analysis thus provided novel and reliable evidence that idiosyncratic semantic category information can be accurately classified by considering the associated brain responses across different individuals.

Importantly, we also explored the anatomical distribution of sensitivity clusters for each category in the intersection analysis. In this analysis, the clusters represent brain regions which contribute to the separation of a given category from the remaining five categories, and thus contain sufficient and specific information for the classifier to identify that particular category. This analysis revealed a category-specific distribution of sensitivity clusters, thus pointing to the existence of segregated representations for each conceptual category within broader anatomo-functional brain regions. Based on the anatomical localization of the sensitivity clusters, we can put forward two main observations. First, we found that each category was sparsely distributed across both hemispheres, and that the distribution of clusters did not fit with the distributed networks which we had hypothesized based on the grounded cognition account. In other words, contrary to the grounded cognition hypothesis, for each given category no specific sensitivity clusters were found within the 
brain regions coding for experiential information relevant for that category. Second, we identified a set of regions that contributed to the distinction of the categories, one from another, namely the inferior frontal gyrus (pars triangularis and pars orbitalis), and the insula, bilaterally. These regions are known to include anatomical and cytoarchitectonic sub-partitions, and have been identified as contributing to multiple cognitive processes (Chang et al., 2012; Amunts et al., 1999; Liakakis et al., 2011). In particular, by combining a large-scale meta-analysis with resting-state connectivitybased parcellation, Chang et al. (2012) showed that the insula is anatomically organized into a tripartite subdivision, with dorsoanterior, ventroanterior, and posterior regions broadly mapping onto cognitive, affective-chemosensory, and sensorimotor processing, respectively. A topographically and functionally targeted meta-analysis including 485 neuroimaging studies (Liakakis et al., 2011) suggested a "multifunctional role" of the inferior frontal gyrus, with the left hemispheric region subserving either verbal language functions, or non-verbal social interaction and empathy functions, and the right hemispheric homologous region related to fine movement control. With respect to our findings, this might indicate that sub-parcels of each anatomical region may code for partially different types of information, possibly based on differential inter-regional connections.

Overall, the results of the multivariate analysis have important implications for theoretical accounts on conceptual knowledge. Firstly, contrary to the embodiment hypothesis that conceptual processing relies on the re-activation of the brain systems coding for experiential information relevant to each semantic category, we found no evidence of the recruitment of the predicted experiential brain systems in sentence processing. The results of the MVPA classification of data across our fMRI localizer study and the sentence processing study indicated that the data of the localizer tasks contained insufficient matching information to allow the discrimination between the six semantic categories at the linguistic semantic level. These results suggest that the functional brain systems activated by direct experience with the conceptual referents of each semantic 
category, (i.e. as identified by the localizer tasks), were not recruited by sentence semantic processing. An alternative interpretation is that, even if the same brain regions were recruited in both the localizer and linguistic tasks, the pattern of neural activity associated with each localizer task differed from the pattern of brain activity associated with processing sentences of the corresponding semantic category. A further possibility is that semantic processing recruits highlevel integrative experiential brain regions rather than the primary experiential brain systems identified by means of our localizer tasks. The latter possibility is supported by a recent fMRI study by Rueschemeyer et al. (2014) that adopted a localizer-based approach for testing the involvement of the motor system in processing action-related meanings. The discrimination between actionrelated versus non-action word categories by the MVPA classifier was only successful in high-level integrative motor areas such as the pre-supplementary motor area and not in primary motor areas. A second implication of the multivariate findings is that, the identification of brain regions (i.e. the left inferior frontal gyrus and the bilateral insula) that contribute to the differentiation of categories from each other points to the existence of regions that, as a whole, are involved in semantic processing, and which nevertheless show an internal category-specific segregation that might rely on a different pattern of anatomical and functional connections with distributed brain regions. This might be consistent with weak embodiment approaches that recognize the existence of conceptual convergence zones in which information from different experiential brain systems is integrated in supra-modal representations (Binder \& Desai, 2011). Alternatively, this is also in line with domainspecific hypotheses that claim for the role of semantic brain regions organized according to different semantic categories (Mahon \& Caramazza, 2008). Future research will need to rely on explicit functional integration semantic models, and employ functional and effective connectivity analyses, to test specifically the hypothesis that different distributed networks based on interregional connections underpin the processing of different semantic categories. This will possibly lead to a refinement of current accounts of conceptual-semantic knowledge.

Finally, the main result of the present study - i.e. the possibility of discriminating each and every 
semantic category at the neural level - informs the need of further developments of the theoretical accounts. While this result underlines the importance of considering meanings along a semantic continuum, over and above the abstract versus concrete distinction, it also emphasizes the need for stronger clarification of the fundamental neural mechanisms underlying the representation of different types of meaning.

The univariate analysis aimed at investigating the neural correlates of the six semantic categories yielded a null result for which strong interpretations are not possible, though it deserves a careful discussion. One possible explanation is that the failure to detect significant effects was due to an inefficient experimental design. However, this interpretation is unlikely in light of the results of the control analysis we performed by defining the activation contrast between experimental and catch trials (section 3.2.1). Increased activation for trials in which participants listened only to sentences, compared to catch trials additionally involving the 1-back task, was found in brain regions broadly covering the perisylvian language network (Friederici, 2012), in the bilateral sensory-motor cortices and in the cingulate gyrus. This result makes it unlikely that an inefficient fMRI event-related design prevented detecting activations in perisylvian, sensory-motor, and limbic cortices that are consistently implicated in language and semantic tasks.

An alternative explanation is that the experimental design inefficiency may specifically concern contrasts assessing distinctions at the semantic level. To address this, we performed a second control analysis (section 3.2.2), exploring the traditional abstract versus concrete distinction, for which an abundant number of studies provide a reference of consolidated evidence (Binder et al., 2009; Wang et al., 2010). The results of the activation contrast tackling this coarser semantic distinction showed that, consistently with previous research (Binder et al., 2009; Wang et al., 2010), processing abstract compared to concrete meanings activated left-hemispheric brain regions, including the pars triangularis in the inferior frontal gyrus, the superior temporal gyrus, and the middle temporal gyrus. Concrete versus abstract sentences processing, in turn, was associated with increased activations in the inferior frontal gyrus and the inferior parietal lobule, which are part of 
the left-hemispheric fronto-parietal circuit for action representation (Pulvermueller \& Fadiga, 2010). On the one hand, these results indicate that our experimental paradigm was capable of revealing semantic distinctions, at least in so far as the traditional abstract-concrete distinction is concerned. On the other hand, however, the involvement of the left-hemispheric action representation circuit in the present study lacked some of the components and subtler features evidenced by previous studies by our group, including the activation of the premotor and anterior parietal regions (Tettamanti et al., 2005) and the activation of the posterior middle temporal gyrus (Tettamanti et al., 2005; 2008). These partial discrepancies call for a thorough examination of other possible shortcomings in our experimental design and, as explained in the following paragraphs, we scrutinised the choice of both the stimuli and the task used in our study.

With respect to the stimuli used, a possible critical issue is the choice of sentences describing actions in a third person perspective, as opposed to the first person perspective employed in our previous studies (Tettamanti et al., 2005; 2008). We can only speculate on the possible impact of pronominal differences, since we did not explicitly manipulate these perspectives within this experiment. Other studies have however previously adopted this approach, providing evidence for motor facilitation for the first versus third person perspective at behavioural level (Gianelli et al., 2011) and by means of transcranial magnetic stimulation (Papeo et al., 2011). Functional MRI investigation showed evidence of increased activity in visual-motion perception areas for first versus third person (Papeo \& Lingnau, 2015), or instead of similarly increased activations in the premotor cortex for both the first and third person (Tomasino et al., 2007). It is difficult to draw strong conclusions in light of the heterogeneity of these effects. However, it does not appear to follow that reduced activations should be expected in the action representation system when using sentences with third person pronouns, as compared to those in the first person.

Another stimulus-related issue to consider is in fact the distinction between multiple fine-grained semantic categories within a single fMRI experiment. Even if we relied on previous evidence that it is possible to clearly distinguish between all the categories under investigation (Ghio et al., 2013), it 
is nevertheless true that some semantic information is shared between categories. For example, sensory and motor semantic information, which is prototypical for action-related categories, also partially extends to Emotion and Mathematics categories. In particular, emotion meanings were rated as including action-related semantic information (Ghio et al., 2013) and emotion word processing activate sensorimotor brain regions (Moseley et al., 2012). Similarly, mathematics meaning ratings were associated with hand action-related semantic information more than with mouth and leg action-related information (Ghio et al., 2013), and a relationship between mathematical concepts and hand finger counting has been suggested (Sato, 2007; Andres, 2008). Previous research showed that overlap and correlation between semantic features can explain semantic effects such as priming effects or specific impairment in patients (for a review, see Vigliocco \& Vinson, 2007). In our experiment, shared features among semantic categories might have determined overlapping activations in some predicted experiential brain areas (e.g., motor areas), such that in these regions the univariate analysis was not sensible enough to detect small between-categories differences, either in spatial extent or in signal change amplitude. While this caveat may account for the lack of significant univariate effects in brain regions activated by semantic features shared between categories, it does not explain the null result in other predicted experiential brain areas which should be activated by semantic features that are unique to a given category (e.g. amygdala for Emotion sentences). In the case of these category-unique representations, we must therefore once again postulate that they are sparse in distributed multivoxel patterns that can be more optimally detected by MVPA than by univariate analyses.

With respect to the 1-back cross-modal task used in the present study, we tried to avoid the shortcomings of passive or minimally demanding tasks (e.g., fixating a point in the visual field), which allow for uncontrolled thoughts with a semantic content (Binder et al., 2009; McKiernan et al., 2006), or the shortcomings of active tasks eliciting a shallow representation of the meaning (e.g., lexical decision), which can be performed by analysing the orthographical or phonological form only (Simmons et al., 2008). In order to elicit a deep meaning processing, explicit semantic 
tasks (e.g., semantic similarity judgements) are often applied, though it turns out that these tasks play a role in modulating semantic processing. For example, Hoenig et al. (2008) showed that the processing of the same semantic categories was associated to activation of either visual or action brain areas, depending whether participants performed a visual- or action-attribute verification task, respectively. The 1-back task used in the present experiment was an active task, which required participants to maintain the phonological form of sentences in short-term memory for a duration sufficient to activate the corresponding semantic representations (Friederici, 2002). Importantly, we did not require any explicit semantic processing of the stimuli, in order to minimize the effect of task on reactivating experiential information associated with the stimuli. It this sense, it is possible that the semantic processing elicited by our task was not sufficiently deep to reactivate detailed and complete semantic information associated with the sentence stimuli. This is a possible consequence that we very carefully considered in designing the study, because we wanted our observations to reflect implicit, automatic semantic processing, rather than post- or meta-semantic judgements tied to a specific task (Kiefer \& Pulvermueller, 2012).

\section{Conclusions}

The present study provided clear-cut evidence that multivariate analysis techniques can reveal the existence of categorically organized information in the patterns of fMRI-measured brain activation along fine-grained semantic distinctions. Our results suggest a degree of commonality across subjects with respect to this semantic organization. These findings contribute to the current debate on conceptual knowledge by suggesting that different types of semantic categories might be associated with specific representational contents as reflected by fine-grained pattern differences of responses sparsely distributed across the cortex. 
Decoding fine-grained conceptual categories

\section{Acknowledgements}

M.G. was supported by a PhD scholarship from the Scuola Normale Superiore, Pisa, Italy. We

thank Karlotta Schlösser for help with the scoring of behavioural responses. We thank three anonymous Reviewers for helpful suggestions on the manuscript. 


\section{References}

Akama, H., Murphy, B., Shimizu, Y., \& Poesio, M. (2012). Decoding semantics across fMRI sessions with different stimulus modalities: a practical MVPA study. Frontiers in Neuroinformatics, 6, 24.

Altarriba, J., Bauer, L. M., \& Benvenuto, C. (1999). Concreteness, context availability, and imageability ratings and word associations for abstract, concrete, and emotion words. Behavior Research Methods, Instruments, \& Computers, 31(4), 578-602.

Amunts, K., Schleicher, A., Bürgel, U., Mohlberg, H., Uylings, H. B., \& Zilles, K. (1999). Broca’s region revisited: cytoarchitecture and intersubject variability. Journal of Comparative Neurology, 412(2), 319-341.

Anderson, A. J., Murphy, B., \& Poesio, M. (2014). Discriminating taxonomic categories and domains in mental simulations of concepts of varying concreteness. Journal of Cognitive Neuroscience, 26(3), 658-681.

Andres M, Olivier E, Badets A (2008) Actions Words and Numbers A Motor Contribution to Semantic Processing? Curr Dir Psychol Sci 17(5): 313-317.

Barsalou, L. W. (2010). Grounded cognition: Past, present, and future. Topics in Cognitive Science, 2(4), 716-724.

Barsalou, L. W., \& Wiemer-Hastings, K. (2005). Situating abstract concepts. In Pecher, D., \& Zwaan, R.A., (Eds.), Grounding Cognition: The role of perception and action in memory, language and thinking. New York: Cambridge University Press, 129-163.

Binder, J. R., \& Desai, R. H. (2011). The neurobiology of semantic memory. Trends in Cognitive Sciences, 15(11), 527-536.

Binder, J. R., Desai, R. H., Graves, W. W., \& Conant, L. L. (2009). Where is the semantic system? A critical review and meta-analysis of 120 functional neuroimaging studies. Cerebral Cortex, 19(12), 2767-2796.

Borghi, A. M., \& Binkofski, F. (2014). Words As social Tools: An embodied view on abstract 
concepts. Berlin; New York, NY: Springer.

Buchweitz, A., Shinkareva, S. V., Mason, R. A., Mitchell, T. M., \& Just, M. A. (2012). Identifying bilingual semantic neural representations across languages. Brain and language, 120(3), 282-289.

Cappa, S. F. (2008). Imaging studies of semantic memory. Current Opinion in Neurology, 21(6), $669-675$.

Carota, F., Moseley, R., \& Pulvermueller, F. (2012). Body-part-specific representations of semantic noun categories. The Journal of Cognitive Neuroscience, 24(6), 1492-1509.

Catricalà, E., Della Rosa, P. A., Plebani, V., Vigliocco, G., \& Cappa, S. F. (2014). Abstract and concrete categories? Evidences from neurodegenerative diseases. Neuropsychologia, 64, 271281.

Chang, L. J., Yarkoni, T., Khaw, M. W., \& Sanfey, A. G. (2012). Decoding the role of the insula in human cognition: Functional parcellation and large-scale reverse inference. Cerebral Cortex, 3, 739-749.

Crutch, S. J., \& Warrington, E. K. (2005). Abstract and concrete concepts have structurally different representational frameworks. Brain, 128(3), 615-27.

Della Rosa, P. A., Catricalà, E., Vigliocco, G., \& Cappa, S. F. (2010). Beyond the abstract-concrete dichotomy: mode of acquisition, concreteness, imageability, familiarity, age of acquisition, context availability, and abstractness norms for a set of 417 Italian words. Behavior Research Methods, 42(4), 1042-1048.

Fox, M. D., \& Raichle, M. E. (2007). Spontaneous fluctuations in brain activity observed with functional magnetic resonance imaging. Nature Reviews Neuroscience, 8(9), 700-711.

Friederici, A. D. (2002). Towards a neural basis of auditory sentence processing. Trends in Cognitive Sciences, 6(2), 78-84.

Friederici, A. D. (2012). The cortical language circuit: from auditory perception to sentence comprehension. Trends in Cognitive Sciences, 16(5), 262-268.

Fusar-Poli, P., Placentino, A., Carletti, F., Landi, P., Allen, P., Surguladze, S., ... Barale, F. (2009). 
Functional atlas of emotional faces processing: a voxel-based meta-analysis of 105 functional magnetic resonance imaging studies. Journal of Psychiatry \& Neuroscience, 34(6), 418.

Gallese, V., \& Lakoff, G. (2005). The Brain's concepts: the role of the sensory-motor system in conceptual knowledge. Cognitive Neuropsychology, 22, 455-479.

Ghio, M., \& Tettamanti, M. (2010). Semantic domain-specific functional integration for actionrelated vs. abstract concepts. Brain and Language, 112(3), 223-232.

Ghio, M. \& Tettamanti, M. (2015). Grounding sentence processing in the sensory-motor system. In L.S. Small \& G. Hickok (eds.), The Neurobiology of Language, Elsevier, 647-657.

Ghio, M., Vaghi, M. M. S., \& Tettamanti, M. (2013). Fine-Grained Semantic Categorization across the Abstract and Concrete Domains. PLoS ONE, 8(6), e67090.

Gianelli, C., Farnè, A., Salemme, R., Jeannerod, M., \& Roy, A. C. (2011). The agent is right: when motor embodied cognition is space-dependent. PLoS ONE, 6(9), e25036.

Gibbs, R. W. (2006). Metaphor Interpretation as Embodied Simulation. Mind \& Language, 21(3), $434-458$.

Granito, C., Scorolli, C., \& Borghi, A. M. (2015). Naming a Lego World. The Role of Language in the Acquisition of Abstract Concepts. PloS one, 10(1), e0114615.

Grezes, J., \& Decety, J. (2001). Functional anatomy of execution, mental simulation, observation, and verb generation of actions: a meta- analysis. Human Brain Mapping, 12(1), 1-19.

Hampton, J. A. (1981). An investigation of the nature of abstract concepts. Memory \& Cognition, $9(2), 149-156$.

Hanke, M., Halchenko, Y., Sederberg, P., Hanson, S., Haxby, J., \& Pollmann, S. (2009). PyMVPA: a Python Toolbox for Multivariate Pattern Analysis of fMRI Data. Neuroinformatics, 7(1), 37-53.

Hanson, S. J., \& Halchenko, Y. O. (2008). Brain reading using full brain support vector machines for object recognition: there is no "face" identification area. Neural Computation, 20(2), 486503.

Haynes, J.-D., \& Rees, G. (2006). Decoding mental states from brain activity in humans. Nature 
Reviews Neuroscience, 7(7), 523-534.

Haxby, J. V., Gobbini, M. I., Furey, M. L., Ishai, A., Schouten, J. L., \& Pietrini, P. (2001). Distributed and overlapping representations of faces and objects in ventral temporal cortex. Science, 293(5539), 2425-2430.

Hoenig, K., Sim, E.-J., Bochev, V., Herrnberger, B., \& Kiefer, M. (2008). Conceptual flexibility in the human brain: dynamic recruitment of semantic maps from visual, motor, and motion-related areas. Journal of Cognitive Neuroscience, 20(10), 1799-1814.

Hoffman, P., Ralph, M. A. L., \& Rogers, T. T. (2013). Semantic diversity: A measure of semantic ambiguity based on variability in the contextual usage of words. Behavior Research Methods, 45(3), 718-730.

Jefferies, E. (2013). The neural basis of semantic cognition: Converging evidence from neuropsychology, neuroimaging and TMS. Cortex, 49(3), 611-625.

Kaplan, J. T., \& Meyer, K. (2012). Multivariate pattern analysis reveals common neural patterns across individuals during touch observation. Neuroimage, 60(1), 204-212.

Kemmerer, D. (2015), Sentence Comprehension. In The Cognitive Neuroscience of Language: An Introduction. Psychology Press.

Kiefer, M., \& Pulvermueller, F. (2012). Conceptual representations in mind and brain: Theoretical developments, current evidence and future directions. Cortex, 48(7), 805-825.

Kousta, S.-T., Vigliocco, G., Vinson, D. P., Andrews, M., \& Del Campo, E. (2011). The representation of abstract words: Why emotion matters. Journal of Experimental Psychology: General, 140(1), 14-34.

Kriegeskorte, N. (2011). Pattern-information analysis: From stimulus decoding to computationalmodel testing. Neuroimage, 56(2), 411-421.

Lambon Ralph, M. A. (2013). Neurocognitive insights on conceptual knowledge and its breakdown. Philosophical Transactions of the Royal Society B: Biological Sciences, 369(1634), 20120392.

Liakakis, G., Nickel, J., \& Seitz, R. J. (2011). Diversity of the inferior frontal gyrus-a meta- 
analysis of neuroimaging studies. Behavioural brain research, 225(1), 341-347.

Mahon, B. Z., \& Caramazza, A. (2008). A critical look at the embodied cognition hypothesis and a new proposal for grounding conceptual content. Journal of Physiology-Paris, 102(1-3), 59-70.

Martin, A. (2007). The Representation of Object Concepts in the Brain. Annual Review of Psychology, 58(1), 25-45.

McKiernan, K. A., D’Angelo, B. R., Kaufman, J. N., \& Binder, J. R. (2006). Interrupting the "stream of consciousness": an fMRI investigation. NeuroImage, 29(4), 1185-1191.

Meteyard, L., Cuadrado, S. R., Bahrami, B., \& Vigliocco, G. (2012). Coming of age: A review of embodiment and the neuroscience of semantics. Cortex, 48(7), 788-804.

Moseley, R., Carota, F., Hauk, O., Mohr, B., \& Pulvermuller, F. (2012). A role for the motor system in binding abstract emotional meaning. Cerebral Cortex, 22(7), 1634-1647.

Mur, M., Bandettini, P. A., \& Kriegeskorte, N. (2009). Revealing representational content with pattern-information fMRI - an introductory guide. Social Cognitive and Affective Neuroscience, 4(1), 101-109.

Nichols, T., Brett, M., Andersson, J., Wager, T., \& Poline, J.-B. (2005). Valid conjunction inference with the minimum statistic. Neuroimage, 25(3), 653-660.

Nieder, A., \& Dehaene, S. (2009). Representation of number in the brain. Annual Review of Neuroscience, 32, 185-208.

Norman, K. A., Polyn, S. M., Detre, G. J., \& Haxby, J. V. (2006). Beyond mind-reading: multi-voxel pattern analysis of fMRI data. Trends in Cognitive Sciences, 10(9), 424-430.

Oldfield, R. C. (1971). The assessment and analysis of handedness: The Edinburgh inventory. Neuropsychologia, 9(1), 97-113.

Olivetti, E., Veeramachaneni, S., \& Nowakowska, E. (2012). Bayesian hypothesis testing for pattern discrimination in brain decoding. Pattern Recognition, 45(6), 2075-2084.

O’Toole, A. J., Jiang, F., Abdi, H., Pénard, N., Dunlop, J. P., \& Parent, M. A. (2007). Theoretical, statistical, and practical perspectives on pattern-based classification approaches to the analysis of 
functional neuroimaging data. Journal of Cognitive Neuroscience, 19(11), 1735-1752.

Paivio, A. (1971). Imagery and verbal processes. New York, NY: Holt, Rinehart \& Winston.

Paivio, A. (2013). Dual coding theory, word abstractness, and emotion: A critical review of Kousta et al. (2011). Journal of Experimental Psychology: General, 142(1), 282-287.

Papeo, L., Corradi-Dell'Acqua, C., \& Rumiati, R. I. (2011). "She" is not like "I": The tie between language and action is in our imagination. Journal of Cognitive Neuroscience, 23(12), 39393948.

Papeo, L., \& Lingnau, A. (2015). First-person and third-person verbs in visual motion-perception regions. Brain and Language, 141, 135-141.

Patterson, K., Nestor, P. J., \& Rogers, T. T. (2007). Where do you know what you know? The representation of semantic knowledge in the human brain. Nature Reviews Neuroscience, $8(12)$, 976-987.

Papeo, L., \& Hochmann, J. R. (2012). A cross-talk between brain-damage patients and infants on action and language. Neuropsychologia, 50(7), 1222-1234.

Penny, W. D., \& Holmes, A. P. (2003). Random Effects Analysis. In K. J. Friston, C. D. Frith, R. Dolan, C. J. Price, S. Zeki, J. Ashburner, ... R. S. J. Frackowiak (Eds.), Human Brain Function (2nd ed., pp. 843-850). San Diego (CA): Academic Press.

Pereira, F., Mitchell, T., \& Botvinick, M. (2009). Machine learning classifiers and fMRI: a tutorial overview. Neuroimage, 45(1), S199-S209.

Pinel, P., Thirion, B., Meriaux, S., Jobert, A., Serres, J., Le Bihan, D., et al., (2007). Fast reproducible identification and large-scale databasing of individual functional cognitive networks. Neuroscience, 8(1), 91.

Poldrack, R. A., Mumford, J. A., Nichols, T. E. (2011). Handbook of functional MRI data analysis. Cambridge University Press.

Pulvermueller, F. (2013). How neurons make meaning: brain mechanisms for embodied and abstract-symbolic semantics. Trends in Cognitive Sciences, 17(9), 458-470. 
Pulvermueller, F., \& Fadiga, L. (2010). Active perception: sensorimotor circuits as a cortical basis for language. Nature Reviews Neuroscience, 11(5), 351-360.

Quadflieg, S., Etzel, J. A., Gazzola, V., Keysers, C., Schubert, T. W., Waiter, G. D., \& Macrae, C. N. (2011). Puddles, parties, and professors: Linking word categorization to neural patterns of visuospatial coding. Journal of Cognitive Neuroscience, 23(10), 2636-2649.

Rueschemeyer, S.-A., Ekman, M., van Ackeren, M., \& Kilner, J. (2014). Observing, performing, and understanding actions: Revisiting the role of cortical motor areas in processing of action words. Journal of Cognitive Neuroscience, 26(8), 1644-1653.

Saccuman, M. C., Cappa, S. F., Bates, E. A., Arevalo, A., Della Rosa, P., Danna, M., \& Perani, D. (2006). The impact of semantic reference on word class: An fMRI study of action and object naming. Neuroimage, 32(4), 1865-1878.

Sato M, Cattaneo L, Rizzolatti G, Gallese V (2007) Numbers within our hands: modulation of corticospinal excitability of hand muscles during numerical judgment. J Cogn Neurosci 19(4): 684-693.

Schrouff, J., Rosa, M. J., Rondina, J. M., Marquand, A. F., Chu, C., Ashburner, J., ... \& MourãoMiranda, J. (2013). PRoNTo: pattern recognition for neuroimaging toolbox. Neuroinformatics, 11(3), 319-337.

Schwanenflugel, P. J., Harnishfeger, K. K., \& Stowe, R. W. (1988). Context availability and lexical decisions for abstract and concrete words. Journal of Memory and Language, 27(5), 499-520.

Simanova, I., Hagoort, P., Oostenveld, R., \& Van Gerven, M. A. (2014). Modality-independent decoding of semantic information from the human brain. Cerebral Cortex, 24(2), 426-434.

Simmons, W. K., Hamann, S. B., Harenski, C. L., Hu, X. P., \& Barsalou, L. W. (2008). fMRI evidence for word association and situated simulation in conceptual processing. Journal of Physiology, Paris.

Simmons, W. K., Reddish, M., Bellgowan, P. S. F., \& Martin, A. (2009). The Selectivity and functional connectivity of the anterior temporal lobes. Cerebral Cortex, 20(4), 813-825. 
Spreng, R. N., Mar, R. A., \& Kim, A. S. N. (2009). The common neural basis of autobiographical memory, prospection, navigation, theory of mind, and the default mode: a quantitative metaanalysis. Journal of Cognitive Neuroscience, 21(3), 489-510.

Tettamanti, M., Buccino, G., Saccuman, M. C., Gallese, V., Danna, M., Scifo, P., et al. (2005). Listening to action-related sentences activates fronto-parietal motor circuits. Journal of Cognitive Neuroscience, 17(2), 273-281.

Tettamanti, M., Manenti, R., Rosa, P. A. D., Falini, A., Perani, D., Cappa, S. F., \& Moro, A. (2008). Negation in the brain: modulating action representations. Neuroimage, 43(2), 358-367.

Tettamanti, M., Rognoni, E., Cafiero, R., Costa, T., Galati, D., \& Perani, D. (2012). Distinct pathways of neural coupling for different basic emotions. Neuroimage, 59(2), 1804-1817.

Tomasino, B., Weiss, P. H., \& Fink, G. R. (2010). To move or not to move: imperatives modulate action-related verb processing in the motor system. Neuroscience, 169(1), 246-258.

Tomasino, B., Werner, C. J., Weiss, P. H., \& Fink, G. R. (2007). Stimulus properties matter more than perspective: An fMRI study of mental imagery and silent reading of action phrases. Neuroimage, 36, T128-T141.

Tottenham, N., Tanaka, J. W., Leon, A. C., McCarry, T., Nurse, M., Hare, T. A., Marcus, D. J., Westerlund, A., Casey, B. J., \& Nelson, C. (2009). The NimStim set of facial expressions: judgments from untrained research participants. Psychiatry research, 168(3), 242-249.

Troche, J., Crutch, S., \& Reilly, J. (2014). Clustering, hierarchical organization, and the topography of abstract and concrete nouns. Frontiers in Psychology, 5, 360.

Vigliocco, G., Kousta, S.-T., Rosa, P. A. D., Vinson, D. P., Tettamanti, M., Devlin, J. T., \& Cappa, S. F. (2014). The Neural Representation of Abstract Words: The Role of Emotion. Cerebral Cortex, 24(7), 1767-1777.

Vigliocco, G., Meteyard, L., Andrews, M., \& Kousta, S. (2009). Toward a theory of semantic representation. Language and Cognition, 1(2), 219-247.

Vigliocco, G., \& Vinson, D. P. (2007). Semantic representation. The Oxford handbook of 
psycholinguistics, 195-215.

Vindiola, M., \& Wolmetz, M. (2011). Mental encoding and neural decoding of abstract cognitive categories: A commentary and simulation. Neuroimage, 54(4), 2822-2827.

Wang, J., Conder, J. A., Blitzer, D. N., \& Shinkareva, S. V. (2010). Neural representation of abstract and concrete concepts: a meta-analysis of neuroimaging studies. Human Brain Mapping, 31(10), 1459-1468.

Wauters, L. N., Tellings, A. E., Van Bon, W. H., \& Van Haaften, A. W. (2003). Mode of acquisition of word meanings: The viability of a theoretical construct. Applied Psycholinguistics, 24(03), 385-406.

Wiemer-Hastings, K., \& Xu, X. (2005). Content differences for abstract and concrete concepts. Cognitive Science, 29(5), 719-736.

Wilson-Mendenhall, C. D., Barrett, L. F., Simmons, W. K., \& Barsalou, L. W. (2011). Grounding emotion in situated conceptualization. Neuropsychologia, 49(5), 1105-1127.

Wilson-Mendenhall, C. D., Simmons, W. K., Martin, A., \& Barsalou, L. W. (2013). Contextual processing of abstract concepts reveals neural representations of nonlinguistic semantic content. Journal of Cognitive Neuroscience, 25(6), 920-935.

Zahn, R., Moll, J., Krueger, F., Huey, E. D., Garrido, G., \& Grafman, J. (2007). Social concepts are represented in the superior anterior temporal cortex. Proceedings of the National Academy of Sciences, 104(15), 6430-6435. 
Table 1. Multivariate classification of fine-grained categories: Bayesian analysis of the confusion matrix. Only the ten highest ranked partitions are shown.

\begin{tabular}{|l|l|}
\hline Bayes Factor & Partition \\
\hline 1 & {$[\mathrm{Ms}],[\mathrm{Em}],[\mathrm{Ma}],[\mathrm{Mo}],[\mathrm{Ha}],[\mathrm{Le}]$} \\
\hline 1.02 & {$[\mathrm{Ms}],[\mathrm{Em}],[\mathrm{Ma}],[\mathrm{Mo}],[\mathrm{Ha}, \mathrm{Le}]$} \\
\hline 2.36 & {$[\mathrm{Ms}, \mathrm{Ma}],[\mathrm{Em}],[\mathrm{Mo}],[\mathrm{Ha}],[\mathrm{Le}]$} \\
\hline 2.40 & {$[\mathrm{Ms}, \mathrm{Ma}],[\mathrm{Em}],[\mathrm{Mo}],[\mathrm{Ha}, \mathrm{Le}]$} \\
\hline 6.21 & {$[\mathrm{Ms}],[\mathrm{Em}],[\mathrm{Ma}, \mathrm{Mo}],[\mathrm{Ha}],[\mathrm{Le}]$} \\
\hline 6.33 & {$[\mathrm{Ms}],[\mathrm{Em}],[\mathrm{Ma}, \mathrm{Mo}],[\mathrm{Ha}, \mathrm{Le}]$} \\
\hline 9.09 & {$[\mathrm{Ms}, \mathrm{Em}],[\mathrm{Ma}],[\mathrm{Mo}],[\mathrm{Ha}],[\mathrm{Le}]$} \\
\hline 9.27 & {$[\mathrm{Ms}, \mathrm{Em}],[\mathrm{Ma}],[\mathrm{Mo}],[\mathrm{Ha}, \mathrm{Le}]$} \\
\hline 22.88 & {$[\mathrm{Ms}],[\mathrm{Em}],[\mathrm{Ma}],[\mathrm{Mo}, \mathrm{Le}],[\mathrm{Ha}]$} \\
\hline 39.00 & {$[\mathrm{Ms}],[\mathrm{Em}],[\mathrm{Ma}],[\mathrm{Mo}, \mathrm{Ha}, \mathrm{Le}]$} \\
\hline
\end{tabular}


Table 2. Multivariate classification of fine-grained categories. Results of the sensitivity analysis with recursive feature elimination applied on each and every pair of categories. An asterisk indicates $\mathrm{p}$-values surviving Bonferroni correction for $\mathrm{n}=15$ comparisons.

\begin{tabular}{lllll}
\hline Pair of categories & Accuracy\%) & Confusion Matrix & Chi-square (p-value) & Bayesian analysis \\
\hline Ms-Em & 66.7 & {$[2715 ; 921]$} & $10.000(0.018)$ & {$[\mathrm{Ms}],[\mathrm{Em}]$} \\
Ms-Ma & 72.2 & {$[3418 ; 218]$} & $28.444(0.000003)^{*}$ & {$[\mathrm{Ms}],[\mathrm{Ma}]$} \\
Ms-Mo & 77.8 & {$[3414 ; 222]$} & $30.222(0.000001)^{*}$ & {$[\mathrm{Ms}],[\mathrm{Mo}]$} \\
Ms-Ha & 83.3 & {$[317 ; 529]$} & $32.222(0.000000)^{*}$ & {$[\mathrm{Ms}],[\mathrm{Ha}]$} \\
Ms-Le & 69.4 & {$[2814 ; 822]$} & $12.888(0.005)$ & {$[\mathrm{Ms}],[\mathrm{Le}]$} \\
Em-Ma & 75.0 & {$[279 ; 927]$} & $18.000(0.0004)^{*}$ & {$[\mathrm{Em}],[\mathrm{Ma}]$} \\
Em-Mo & 81.9 & {$[307 ; 629]$} & $29.444(0.000002)^{*}$ & {$[\mathrm{Em}],[\mathrm{Mo}]$} \\
Em-Ha & 80.6 & {$[297 ; 729]$} & $26.888(0.000006)^{*}$ & {$[\mathrm{Em}],[\mathrm{Ha}]$} \\
Em-Le & 70.8 & {$[2510 ; 1126]$} & $12.555(0.006)$ & {$[\mathrm{Em}],[\mathrm{Le}]$} \\
Ma-Mo & 73.6 & {$[2710 ; 926]$} & $16.111(0.001)^{*}$ & {$[\mathrm{Ma}],[\mathrm{Mo}]$} \\
Ma-Ha & 86.1 & {$[293 ; 733]$} & $38.444(0.000000)^{*}$ & {$[\mathrm{Ma}],[\mathrm{Ha}]$} \\
Ma-Le & 69.4 & {$[228 ; 1428]$} & $12.888(0.005)$ & {$[\mathrm{Ma}],[\mathrm{Le}]$} \\
Mo-Ha & 73.6 & {$[225 ; 1431]$} & $20.555(0.000130)^{*}$ & {$[\mathrm{Mo}],[\mathrm{Ha}]$} \\
Mo-Le & 66.7 & {$[219 ; 1527]$} & $10.000(0.018)$ & {$[\mathrm{Mo}],[\mathrm{Le}]$} \\
Ha-Le & 65.3 & {$[2514 ; 1122]$} & $7.220(0.065)$ & {$[\mathrm{Ha}],[\mathrm{Le}]$} \\
\hline
\end{tabular}




\section{Figure legends}

Figure 1. A. Confusion matrix of the multivariate classification of fine-grained categories, showing the number of correctly (diagonal) and incorrectly (off-diagonal) classified participants. B. Category-specific sensitivity intersection maps, representing the intersection of the five sensitivity maps between one particular category and each of the other five categories. All effects shown are displayed on cortical renderings and on axial ( $\mathrm{z}$ coordinate levels in $\mathrm{mm}$ ) slices of the anatomical image of one of the participants (warped to the MNI coordinate space). 
Figure 1
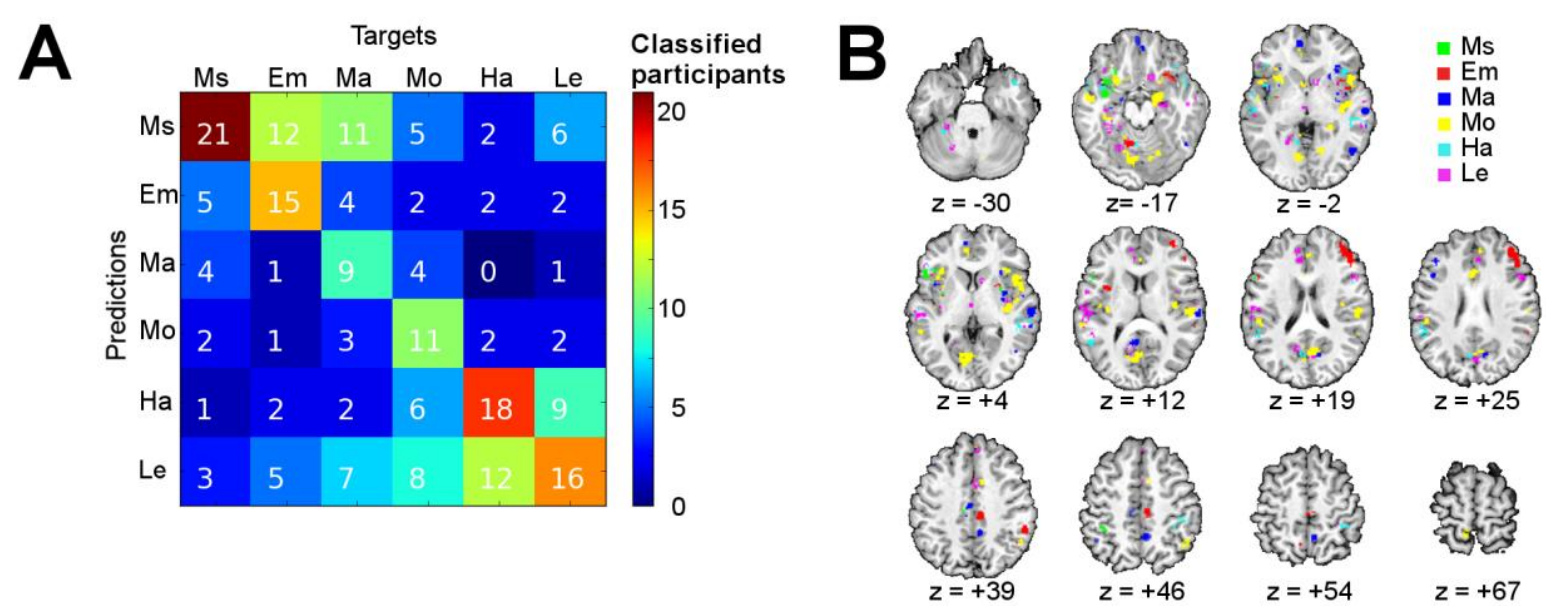

10. Supplementary Material
Click here to download $10 . \mathrm{S}$

10. Supplementary Material
Click here to download 10. Supplementary Material: GhioMetal_supplmat_RevIII_2feb2016.pdf

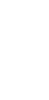

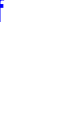

